\title{
Responding to Unfair Offers Made by a Friend: Neuroelectrical Activity Changes in the Anterior Medial Prefrontal Cortex
}

\author{
Camila Campanhã, ${ }^{1}$ Ludovico Minati, ${ }^{2}$ Felipe Fregni, ${ }^{3,4}$ and Paulo S. Boggio ${ }^{1}$ \\ ${ }^{1}$ Social and Cognitive Neuroscience Laboratory and Developmental Disorders Program, Center for Health and Biological Sciences, Mackenzie Presbyterian \\ University, 01241-001, São Paulo, Brazil, ${ }^{2}$ Scientific Department, Fondazione IRCCS Istituto Neurologico “Carlo Besta," 20133, Milano, Italy, ${ }^{3}$ Laboratory of \\ Neuromodulation, Department of Physical Medicine and Rehabilitation, Spaulding Rehabilitation Hospital and Massachusetts General Hospital, Harvard \\ Medical School, Boston, Massachusetts 02114, and ${ }^{4}$ Department of Neurology, Beth Israel Deaconess Medical Center, Harvard Medical School, Boston, \\ Massachusetts 02115
}

When receiving unfair monetary offers from another person, the most common response is punishment. Existing literature on the Ultimatum Game indicates that individuals frequently refuse unfair offers even when this results in a loss for themselves. Here, we present behavioral and neurophysiological evidence demonstrating that friendship substantially modifies this response. When the proposer was a friend rather than an unknown person, unfair offers were less frequently rejected and the medial frontal negativity (MFN) typically associated with unfair offers was reversed to positive polarity. The underlying generators were located in inferior-mesial and right inferior- and medial-lateral frontal regions (BA10 and BA11). These findings highlight the fundamental role of the anterior prefrontal cortex in interpersonal economic interaction and, particularly, present new evidence on the effects of social distance on the MFN.

\section{Introduction}

When making a decision faced with unfairness, we tend to punish others even when this results in a loss for ourselves (Fehr and Gächter, 2002). From an evolutionary perspective, such strategy is understandable, as it minimizes the probability of similar future harm. This process, which is also present in nonhuman primates, appears to be related to emotional processing and is associated with activation of reward-related networks (de Quervain et al., 2004; Brosnan and De Waal, 2003).

Social decision making has received considerable attention in recent years. The Ultimatum Game (UG) (Güth et al., 1982) is a widely used scenario where a proposer offers to divide money between herself and another player. When the recipient accepts, both gain; when they do not accept, neither person receives anything. Recipients typically reject about half of the unfair offers (80:20 or 90:10 ratio). This response is associated with a specific neuroelectric signature, consisting of a larger medial frontal neg-

Received March 11, 2011; revised Sept. 6, 2011; accepted Sept. 12, 2011.

Author contributions: C.C. and P.S.B. designed research; C.C. and P.S.B. performed research; C.C., L.M., F.F., and P.S.B. analyzed data; C.C., L.M., F.F., and P.S.B. wrote the paper.

C.C. was supported by Fundação de Amparo à Pesquisa do Estado de São Paulo (2009/04694-1). P.S.B. was supported by Conselho Nacional de Desenvolvimento Científico e Tecnológico (305718/2009-6) and by Mack Pesquisa. We acknowledge Kayleen Weaver for proofreading of this manuscript, Nathalia I. Baptista for picture design, and two anonymous reviewers for insightful feedback on an earlier version.

The authors declare no competing financial interests.

Correspondence should be addressed to Dr. Paulo Sérgio Boggio, Social and Cognitive Neuroscience Laboratory and Developmental Disorders Program, Center for Health and Biological Sciences, Mackenzie Presbyterian University, Rua Piaui, 181, $10^{\circ}$ andar, Sao Paulo, SP, 01241-001, Brazil. E-mail: boggio@mackenzie.br.

DOI:10.1523/JNEUROSCI.1253-11.2011

Copyright $\odot 2011$ the authors $\quad 0270-6474 / 11 / 3115569-06 \$ 15.00 / 0$ ativity (MFN) component (Boksem and De Cremer, 2010; Hewig et al., 2011).

An open question is whether punishment of unfair offers might be affected by the relationship one has with the proposer, such as when an offer comes from a close friend. Despite the fundamental role that friendship plays in all domains of life, very little is known on how it impacts decision-making processes. In this study, participants played an adapted version of the Ultimatum Game, believing that they were facing two proposers: an accompanying friend and an unknown person. Participants were asked to take with them a friend to the experimental laboratory. The participant/friend pair was allowed to participate depending on the level of trust between them. Here, we present behavioral and neurophysiological evidence demonstrating that friendship profoundly modulates the way we process and respond to fair and unfair offers.

\section{Materials and Methods}

Participants. Fifteen healthy volunteers ( 7 male, mean age $21.5 \pm 2.3$, range 18-25) participated in the study, which was approved by the institutional ethics committee of the Mackenzie Presbyterian University, Brazil and by the National Ethics Committee (SISNEP, Brazil), and informed consent was obtained from all subjects.

Procedure. Participants were instructed to come to the laboratory accompanied by a friend, as they would receive offers from him/her. Participants would also receive offers from an unknown person, introduced to them upon arrival. The gender ratio was matched between friends and unknown proposers, and there was no difference in age among proposer and active participants. To guarantee the same level of reciprocal trust, we administered an adapted version of the KnowI scale (Turan and Horowitz, 2007), in which references to romantic relationship were re- 
a

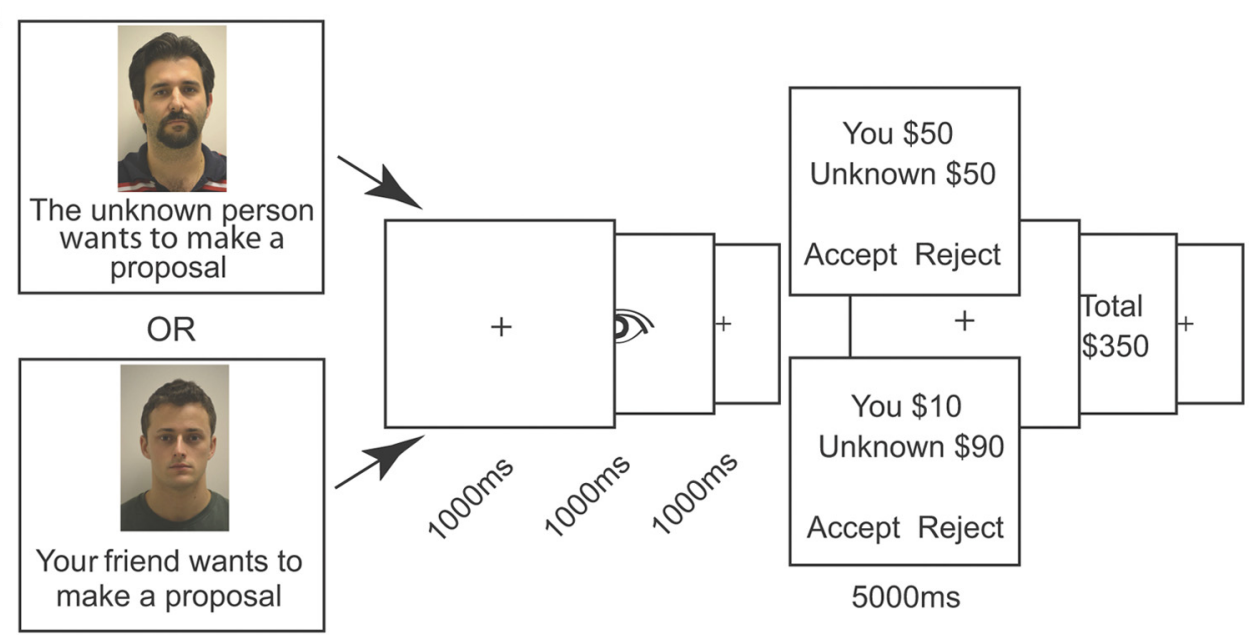

$1500 \mathrm{~ms}$

b Judgment of proposer's fairness

C Rate of Acceptance
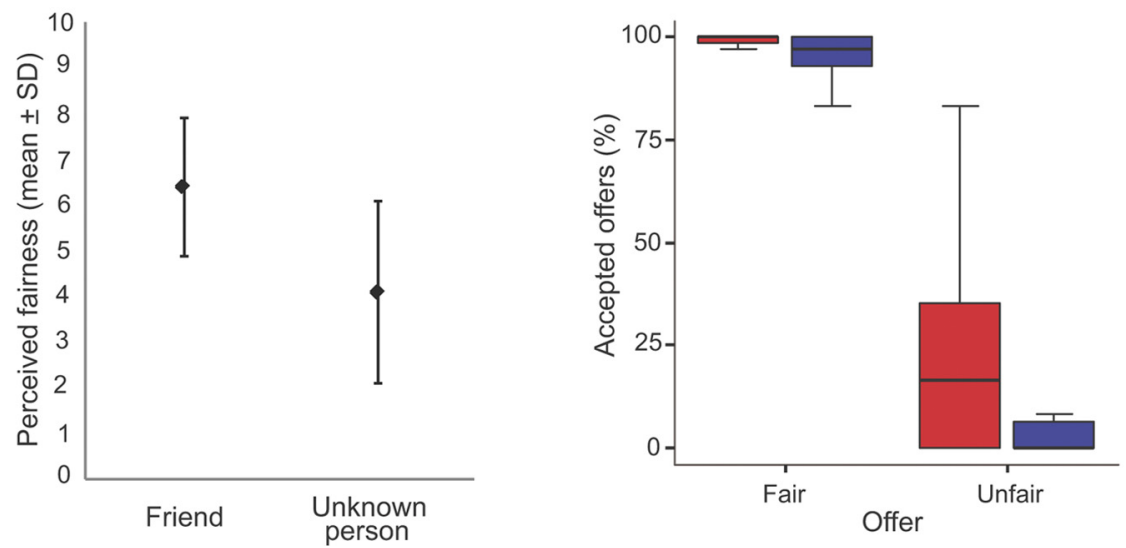

Figure 1. Experimental paradigm and behavioral data. $\boldsymbol{a}$, Participants played the Ultimatum Game with a friend and with an unknown person. In each trial, the surrogate proposer appeared first, followed by a prompt to blink if necessary. A fair or unfair monetary offer was then presented. After accepting or rejecting, the updated balance was shown. $\boldsymbol{b}$, Participants rated their friend as overall fairer than the unknown proposer ( $p<0.001$, bars denote SD). $c$, Throughout the game, participants accepted more fair $(p=0.03$, stem-and-leaf plot) and unfair ( $p=0.006)$ offers believed to originate from their friend than from the unknown proposer.

worded to refer to friendship. Participants were recruited only if they and their friend had a score $>150$. None of the participants had been engaged in a relationship with the accompanying friend, or were related to them. There was no significant difference in the resulting reciprocal trust scores. Since previous work has demonstrated that the Moral Identity score (Aquino and Reed, 2002) correlates with UG performance (Boksem and De Cremer, 2010), we recruited participants in the midrange (25-37) to limit interindividual variability and exclude those with very high and low values.

Ultimatum Game task. Throughout the game, participants were presented with proposals from three categories: fair (50:50 ratio), moderately unfair (70:30), and unfair (80:20 or 90:10), which were randomly chosen by the computer without any human interaction. Before playing, they were not made aware that proposals would be drawn from these categories, and that there would be no offers more advantageous for the recipient than the proposer. For each trial, the picture of the surrogate proposer appeared first (either friend or unknown person), followed by the monetary offer and a forced-choice prompt (Fig. 1a). After response, the updated balance was displayed. The proposer's balance was never shown. For each proposer, 72 stimuli were delivered in a counterbalanced order, for a total of 144 trials. The sequence of proposers (friend vs unknown person) was randomized across subjects. Upon task completion, participants rated the perceived fairness of the proposers through a visual analog scale $(0=$ very unfair to $10=$ very fair). They were subsequently asked whether they believed that they were really playing with their friend and with an unknown person, rather than with the computer. Due to local ethics restrictions, participants could not receive any fraction of their earnings. However, they were told that at the end of the whole study, the highest-performing one would receive a gift card, and where applicable, they were awarded university credits for experiment participation.

EEG recording. To investigate the neural correlates of the observed behavioral changes, we recorded the electroencephalogram using a 128 geodesic sensor net (Electrical Geodesics). Following artifact rejection and re-referencing to average, the signals were epoched in the $-200 \mathrm{~ms}$ to $800 \mathrm{~ms}$ interval with respect to offer presentation. For each proposer, the difference waveform between unfair and fair proposals was generated to reveal the MFN component (Boksem and De Cremer, 2010), which was evaluated on the left, right, and medial frontal regions (F4-Fz-F3, FC4-FCz-FC3 sites). An averaging window between 200 and $330 \mathrm{~ms}$ was defined on the basis of the grand average wave. Before averaging, we excluded epochs contaminated by eyeblink, ocular movement, or other artifacts. Epochs containing a difference $>140 \mu \mathrm{V}$ between channels above and below the eyes, a difference $>55 \mu \mathrm{V}$ between channels near the outer canthi, or one or more channels exceeding an amplitude of $200 \mu \mathrm{V}$ were automatically rejected. The remaining epochs were manually reviewed by an experienced operator, who removed those containing residual artifact. Overall, $\sim 15 \%$ of epochs were rejected. 


\section{Results}

Nonparametric Wilcoxon tests comparing offer acceptance rate according to fairness and friendship indicated that participants accepted more fair (median 100\%, range $89-100 \%$ vs median $97 \%$, range $81-100 \% ; Z=-2.2, p=0.03$ ) and unfair (median $17 \%$, range $0-83 \%$ vs median $0 \%$, range $0-42 \% ; Z=-2.8, p=$ $0.006)$ offers believed to originate from their friend rather than from the unknown proposer. There was no significant difference between friend and unknown proposer in the acceptance of moderately unfair offers $(30: 70 ; p=0.3)$. Overall, participants perceived their friend to be fairer than the unknown person as indexed by their post-UG visual analog scale scores $(6.4 \pm 1.5$ vs $4.1 \pm 1.9, t_{(14)}=4.5, p<0.001$ ) (Fig. 1b,c). When assessed at debriefing, all participants believed that they were really playing with their friend and with the unknown person, rather than with the computer.

A repeated-measures ANOVA was also performed to analyze reaction times with respect to friendship and response (accepted fair offers vs rejected unfair offers). The reaction times of accepted unfair offers and rejected fair offers were not analyzed as they were under-represented. There was no effect of friendship and no interaction between the two factors, but there was a significant main effect of response $\left(F_{(1,14)}=28.8 ; p<0.001\right)$, with overall faster acceptance of fair offers than rejection of unfair offers (927 $\pm 340 \mathrm{~ms}$ vs $1140 \pm 370 \mathrm{~ms}$ ).

With regards to the MFN potential, we performed a repeated-measures ANOVA analyzing the difference between unfair and fair proposals with respect to friendship and electrode site (left frontal, right frontal, or midline). KolmogorovSmirnov tests were performed to confirm that the data were normally distributed. We found a significant main effect of friendship, i.e., a difference between friend and unknown person (respectively, $1.6 \pm 1.9 \mu \mathrm{V}$ vs $-1.7 \pm 1.7 \mu \mathrm{V} ; F_{(1,14)}=$ $\left.19.5, p=0.001, \eta_{\mathrm{p}}^{2}=0.6\right)$; there was no effect of electrode site and no interaction between the two factors. Subsequent onesample $t$ tests confirmed that the offers from the friend elicited a significant positivity, whereas those from the unknown proposer elicited a significant negativity $\left(t_{(14)}=3.2, p=0.002\right.$ and $t_{(14)}=-3.9, p=0.007$, respectively; Fig. $\left.2 a, b\right)$. We additionally analyzed the difference between moderately unfair and fair proposals, and did not find any effect of friendship, electrode site, or interaction.

Source localization was performed using standardized LowResolution Brain Electromagnetic Tomography (sLORETA) (Pascual-Marqui et al., 1994). Cortical current density maps for the group-averaged MFN are shown in Figure $2 c$. Proposals from the unknown proposer elicited an intense negative current difference in inferior-mesial and right inferior-lateral frontal regions. Proposals from the friend were associated with a similar pattern of opposite polarity. Statistical significance maps for the comparison between the two proposers were generated through voxelby-voxel paired $t$ tests. Accounting for multiple comparisons, we set $\alpha=0.001$, corresponding to a critical $t$ value of 4.1 . Significantly more negative current difference was detected for offers from the unknown person in 42 voxels (peak $t$ score $4.7, p<$ $0.001)$, clearly clustered in inferior-mesial and right inferior- and medial-lateral frontal regions (80\% within the right Brodmann area $11,20 \%$ within the right BA 10). The effect did not reach the corrected significance threshold for any voxel in the left hemisphere, and the converse difference was not observed for any region.

\section{Discussion}

A high rejection ratio was observed for unfair offers coming from the unknown proposer, confirming previous findings on inequity aversion and punishment of injustice in social interactions where the proposer is not known. This behavior stimulates communal fairness and altruism (Fehr and Gächter, 2002; Brosnan and De Waal, 2003). Our behavioral data indicate enhancement of utilitarian behavior when playing with a friend, i.e., an attenuation of altruistic punishment. In principle, this could be explained as a framing effect, in which friendship provokes a fundamental bias affecting the subjective perception of offer fairness, reflected in acceptance rates and proposer fairness ratings. Higher acceptance could also indicate an expectation that initial unfairness will be offset later in the game. Another explanation is that the participant sees no reason to altruistically punish their friend, and prioritizes personal gain as a consequence.

Similarly, it is possible that the participant attaches a certain subjective value to friendship, and wishes to avoid damaging it by sending a negative judgment of unfairness. This would be a longterm strategy in which the participants modulate their punishment responses as a function of the proposer targeting potential future gains. In this perspective, the species-wide benefit of extinguishing a maladaptive behavior would prevail when playing with unknown proposers, but be suppressed when playing with a friend to prevent offending them. In this direction, it is important to highlight that our findings are in agreement with and extend a previous study (Moore, 2009) showing that children share more with their friends than with others in a zero-sum game.

It is important to consider that the UG is typically played as a one-round game, implying that rejection cannot be due to selfinterest as the proposer will have no chance to make new offers to a given recipient (van Dijk et al., 2004). In our experiment, however, the UG was played in several rounds with the same proposer/ recipient pair. Thus, rejections might reflect bargaining behavior based on self-interest, and not only a response to offer fairness. At the same time, recipients played with a close friend and with an unknown person. With this, social distance might be an important variable to explain our results.

For instance, previous studies have already shown that identification increases cooperation on games such as Dictator Game and Prisoner's Dilemma as it decreases social distance (Frey and Bohnet, 1997; Bohnet and Frey, 1999). In our study, social distance combined with multiple rounds might have resulted in responses driven by a combination of retaliation, gain-related strategic behavior (when the proposer was the unknown person), and avoidance of subsequent conflict (when the proposer was the friend) [see Handgraaf et al. (2003) for a review on social utility in ultimatum bargaining]. Further, it is a well established notion that the expectation and prevention of future regret influences present decision making (Larrick and Boles, 1995; Zeelenberg et al., 1996; Zeelenberg and Beattie, 1997), and the fact that participants believed that they were playing with their friend might have promoted the acceptance of unfair offers as a way of minimizing the expectation of regret related to potential disappointment and conflict after the end of the game.

Our ERP findings are in agreement with the study by Boksem and De Cremer (2010), in which the amplitude of the MFN, peaking around $260 \mathrm{~ms}$ over frontal sites, indexed the perceived level of offer fairness (more pronounced for unfair offers), and was modulated by the participant's level of concern for fairness. Similarly to the present experiment, in their study participants played multiple rounds of the game with each proposer. Our data 
a

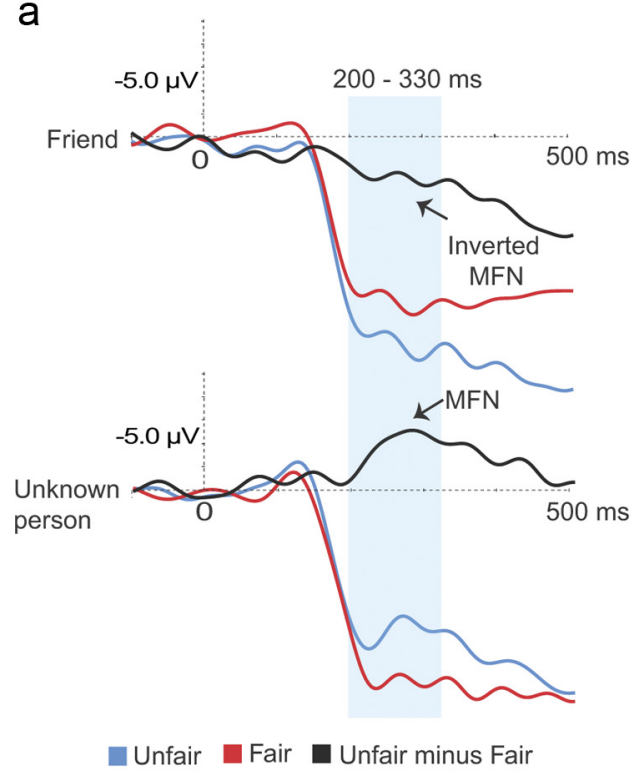

b

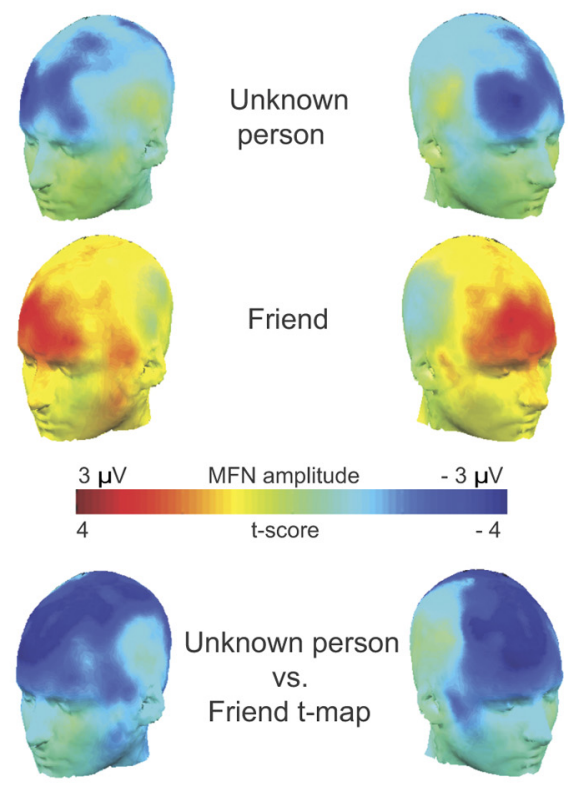

\section{C}

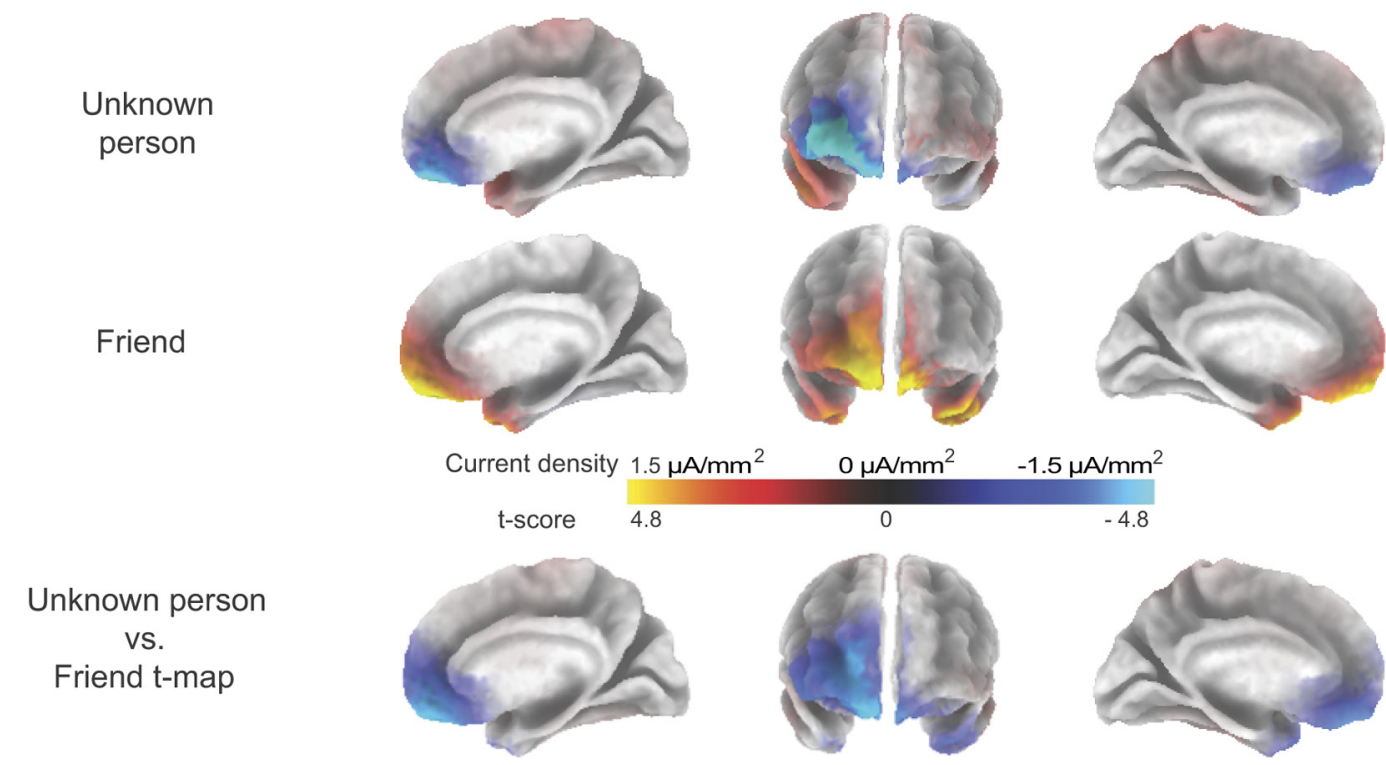

Figure 2. Electrophysiological data. $\boldsymbol{a}$, Offers from the unknown proposer evoked an MFN component, defined as the difference in neuroelectric response to fair and unfair offers. For offers from the friend, the polarity was reversed $(p=0.001) \cdot \boldsymbol{b}$, The topographies present (unfair minus fair in unknown person), (unfair minus fair in friend), and [(unfair minus fair in unknown person) minus (unfair minus fair in friend)]. The MFN difference between proposers was prominent bilaterally over central anterior-frontal scalp regions. c, Source localization present (unfair minus fair in unknown person), (unfair minus fair in friend), and [(unfair minus fair in unknown person) minus (unfair minus fair in friend)]. Source localization revealed that the underlying cortical generators were in inferior-mesial and right inferior- and medial-lateral frontal regions, i.e., within Brodmann areas 10 and 11.

extend their results by demonstrating that the MFN is strongly responsive to social distance, reversing polarity when the proposer is a close friend rather than an unknown person. The MFN is generally assumed to originate from the anterior cingulate cortex (Gehring and Willoughby, 2002). However, the inverse solution in our case revealed engagement of anterior structures of the medial prefrontal cortex (MPFC). Specifically, the comparison between the two proposers revealed more negative current difference for offers from the unknown person within BA10 and BA11. This discrepancy may indicate that, as found with many mid- and long-latency components, the underlying cortical generators involve a network of regions, whose relative contribution on the inverse reconstruction maps may vary as a function of the specific task features and stability of the solution. It may also more simply be a consequence of inverse solution instability and represent activity actually originating from BA24/32.

Broadly, it has been shown that BA10 and BA11 are engaged during tasks involving cooperation in two-person decision-making games (McCabe et al., 2001), and subserve person perception as well as mentalization (for review, see Amodio and Frith, 2006). As confirmed in a meta-analysis by Steele and Lawrie (2004), these regions are also crucially related to emotion. In our task, efforts to understand the other's intentions and motives (Frith and Frith, 2003; Frith and Singer, 2008) might have had an important role in driving activity in BA10 and BA11 and might have guided the behavioral responses. Indeed, as proposed by Amodio and Frith (2006), the anterior MFC can play a role in "meta-cognitive processes of reflecting on feelings and intentions." 
More specifically, previous experiments point to a close relationship between the MFN and dopaminergic activity. In the studies by Holroyd et al. (2004) and Nieuwenhuis et al. (2004), undesirable outcomes were associated with strongest negativity, while positive outcomes elicited weaker MFN responses. In parallel, converging results indicate that unexpected rewards elicit positive responses in the MFN time window (Potts et al., 2006; Holroyd et al., 2008, 2011; Pfabigan et al., 2011). Together, these findings closely follow the known pattern of dopaminergic system response to reward and reward prediction error. In fact, it was previously proposed by Holroyd and Coles (2002) that frontal negative responses may represent increased deep-layer cortical activity due to attenuated inhibitory dopaminergic input. By the same logic, a positive scalp potential could result from deeplayer inhibition during enhanced dopamine release, e.g., reward delivery.

From this perspective, our results would imply that unfair offers dampen dopaminergic activity with respect to fair offers when originating from an unknown person, but boost it when made by a friend. This effect cannot be explained in terms of altruistic punishment: our behavioral data indicate more pronounced altruistic punishment when playing against an unknown person, and the well established notion that altruistic punishment is associated with increased dopaminergic activity (e.g., Strobel et al., 2011) would lead to predicting the opposite difference. An alternative account is that, as proposed by Boksem et al. (2011a), the MFN may represent an abstract marker of "social pain," i.e., may reflect negative outcomes of a social nature such as unfair treatment by others. For example, Boksem et al. (2011a) evaluated the MFN during a game in which the player's performance did not affect the other participant (an unknown person), and reported that the MFN was largest in trials during which the participant lost and the other player won, leading to the idea that the MFN might index the comparison between one's and other people's experienced outcomes, i.e., fairness related to social structures (Handgraaf et al., 2003). Since the unfair offers originating from the friend were perceived as less unfair than those from the unknown person, the difference in "social pain" might have reflected in MFN amplitude. Speculatively, this account might even explain the observed inversion in polarity: if receiving an unfair offer from an unknown person is more "painful" (i.e., deactivates the dopaminergic system more strongly) than receiving it from a friend, once average reward expectations have built up over a few trials, the dopaminergic system may generate a positive reward prediction error signal for the less "painful" situation of receiving an unfair offer from the friend and a negative signal for more "painful" unfair offers by the unknown person.

Clearly, one limitation of this study is that the experimental comparison, i.e., friend versus unknown person, contained multiple elements such as social distance, similarity, sympathy, and trustfulness. As a consequence, we are unable to directly map the observed effects to a specific factor. Further work is necessary to try to disentangle the effects of these individual factors, for example by isolating knowledge, degree of sympathy, etc. with specific regressions; this is likely to require a much larger population to attain adequate statistical power. Another limitation is the absence of real monetary endowment. While we cannot exclude that this may have introduced differences with respect to experiments in which financial reward was available, other studies in this field have obtained consistent findings without paying participants (e.g., Polezzi et al., 2008; Boksem et al., 2011b), and it has been established that small monetary incentives do not nec- essarily boost task performance and may even have paradoxical effects (Gneezy and Rustichini, 2000).

More than two millennia ago, Aristotle wrote: “... we prize friendship for itself, even though nothing else is likely to come to us from it." Our data demonstrate that this tendency has a neurobiological correlate at the level of anterior MPFC engagement, likely reflecting dopaminergic system activity, which accompanies the strong behavioral effects observed during a social decision-making task. There are fundamental differences in the way we process offers coming from a friend or an unknown person. These can be either seen as beneficial, since unfair offers do not elicit a punishment response among friends, or as socially maladaptive, as we become lenient about unfair proposals. Further experiments are needed to disentangle the exact dynamics underlying the observed behavioral and neuroelectric effects, in particular to verify whether the MFN amplitude can be modeled as a reward prediction error signal in the context of "social pain."

\section{References}

Amodio DM, Frith CD (2006) Meeting of minds: the medial frontal cortex and social cognition. Nat Rev Neurosci 7:268-277.

Aquino K, Reed A 2nd (2002) The Self-Importance of Moral Identity. J Pers Soc Psychol 83:1423-1440.

Bohnet I, Frey BS (1999) The sound of silence in prisoner's dilemma and dictator games. J Econ Behav Organ 38:43-57.

Boksem MAS, De Cremer D (2010) Fairness concerns predict medial frontal negativity amplitude in ultimatum bargaining. Soc Neurosci 5:118-128.

Boksem MAS, Kostermans E, De Cremer D (2011a) Failing where others have succeeded: medial frontal negativity tracks failure in a social context. Psychophysiology 48:973-979.

Boksem MAS, Ruys KI, Aarts H (2011b) Facing disapproval: performance monitoring in a social context. Soc Neurosci 6:360-368.

Brosnan SF, De Waal FB (2003) Monkeys reject unequal pay. Nature 425:297-299.

de Quervain DJ, Fischbacher U, Treyer V, Schellhammer M, Schnyder U, Buck A, Fehr E (2004) The neural basis of altruistic punishment. Science 305:1254-1258.

Fehr E, Gächter S (2002) Altruistic punishment in humans. Nature 415:137-140.

Frey BS, Bohnet I (1997) Identification in democratic society. J Socio Econ 26:25-38.

Frith CD, Singer T (2008) The role of social cognition in decision making. Philos Trans R Soc Lond B Biol Sci 363:3875-3886.

Frith U, Frith CD (2003) Development and neurophysiology of mentalizing. Philos Trans R Soc Lond B Biol Sci 358:459-473.

Gehring WJ, Willoughby AR (2002) The medial frontal cortex and the rapid processing of monetary gains and losses. Science 295:2279-2282.

Gneezy U, Rustichini A (2000) Pay enough or don't pay at all. Q J Econ 115:791-810.

Güth W, Schmittberger R, Schwarze B (1982) An experimental analysis of ultimatum bargaining. J Econ Behav Organ 3:367-388.

Handgraaf MJJ, Van Dijk E, De Cremer D (2003) Social utility in ultimatum bargaining. Social Justice Res 16:263-283.

Hewig J, Kretschmer N, Trippe RH, Hecht H, Coles MG, Holroyd CB, Miltner WH (2011) Why humans deviate from rational choice. Psychophysiology 48:507-514.

Holroyd CB, Coles MGH (2002) The neural basis of human error processing: reinforcement learning, dopamine, and the error-related negativity. Psychol Rev 109:679-709.

Holroyd CB, Larsen JT, Cohen JD (2004) Context dependence of the eventrelated brain potential associated with reward and punishment. Psychophysiology 41:245-253.

Holroyd CB, Pakzad-Vaezi KL, Krigolson OE (2008) The feedback correctrelated positivity: sensitivity of the event-related brain potential to unexpected positive feedback. Psychophysiology 45:688-697.

Holroyd CB, Krigolson OE, Lee S (2011) Reward positivity elicited by predictive cues. Neuroreport 22:249-252.

Larrick RP, Boles TL (1995) Avoiding regret in decisions with feedback: a negotiation example. Organ Behav Hum Decis Process 63:87-97. 
McCabe K, Houser D, Ryan L, Smith V, Trouard T (2001) A functional imaging study of cooperation in two-person reciprocal exchange. Proc Natl Acad Sci U S A 98:11832-11835.

Moore C (2009) Fairness in children's resource allocation depends on the recipient. Psychol Sci 8:944-948.

Nieuwenhuis S, Yeung N, Holroyd CB, Schurger A, Cohen JD (2004) Sensitivity of electrophysiological activity from medial frontal cortex to utilitarian and performance feedback. Cereb Cortex 14:741-747.

Pascual-Marqui RD, Michel CM, Lehmann D (1994) Low resolution electromagnetic tomography: a new method for localizing electrical activity in the brain. Int J Psychophysiol 18:49-65.

Pfabigan DM, Alexopoulos J, Bauer H, Sailer U (2011) Manipulation of feedback expectancy and valence induces negative and positive reward prediction error signals manifest in event-related brain potentials. Psychophysiology 48:656-664.

Polezzi D, Lotto L, Daum I, Sartori G, Rumiati R (2008) Predicting outcomes of decisions in the brain. Behav Brain Res 187:116-122.

Potts GF, Martin LE, Burton P, Montague PR (2006) When things are better or worse than expected: the medial frontal cortex and the allocation of processing resources. J Cogn Neurosci 18:1112-1119.

Steele JD, Lawrie SM (2004) Segregation of cognitive and emotional function in the prefrontal cortex: a stereotactic meta-analysis. Neuroimage 21:868-875.

Strobel A, Zimmermann J, Schmitz A, Reuter M, Lis S, Windmann S, Kirsch $P$ (2011) Beyond revenge: neural and genetic bases of altruistic punishment. Neuroimage 54:671-680.

Turan B, Horowitz LM (2007) Can I count on you to be there for me? Individual differences in a knowledge structure. J Pers Soc Psychol 93:447-465.

van Dijk E, De Cremer D, Handgraaf MJJ (2004) Social value orientations and the strategic use of fairness in ultimatum bargaining. J Exp Soc Psychol 40:697-707.

Zeelenberg M, Beattie J (1997) Consequences of regret aversion 2: additional evidence for effects of feedback on decision making. Organ Behav Hum Decis Process 72:63-78.

Zeelenberg M, Beattie J, Van Der Pligt J, De Vries NK (1996) Consequences of regret aversion: effects of expected feedback on risky decision making. Organ Behav Hum Decis Process 65:148-158. 